\title{
Underwater Wireless Power Transfer for Maritime Applications
}

\author{
Mohammed Sabith ${ }^{1}$, Muhammad Sayed ${ }^{2}$, Nihal K ${ }^{3}$, Geethu James ${ }^{4}$ \\ 1,2,3 Student, Dept. of Electrical and Electronics Engineering, Mar Athanasius College of Engineering, \\ Kothamangalam, Kerala, India \\ ${ }^{4}$ Assistant Professor, Dept. of Electrical and Electronics Engineering, Mar Athanasius College of Engineering, \\ Kothamangalam, Kerala, India
}

\begin{abstract}
For Unmanned Underwater Vehicles (UUVs), power is the critical factor that often determines mission lifetime. Unlike many terrestrial vehicles, where batteries can be easily replaced or recharged, UUVs must either surface for battery replacement, or make watertight connections to safely recharge. This project presents the development and implementation of an inductive, underwater wireless power transfer system for use with unmanned underwater vehicles (UUVs). Specially, the design and fabrication of power transfer coils and power electronics is provided for a system capable of providing $75 \mathrm{~W}$ to a load. At small stand-of distances ( $<2$ inches) and frequencies below 300 $\mathrm{kHz}$, it is shown that there is little difference between inductive power transfer in air and seawater. Measured data shows that at power levels of $75 \mathrm{~W}$, the system efficiency from the transmitter to a receiver and resistive load is above $85 \%$.
\end{abstract}

Key Words: Wireless Power Transfer (WPT), Transmitter (Tx), Receiver (Rx), Relative Permeability $\left(\mu_{r}\right)$, Relative Permittivity $\left(\epsilon_{r}\right)$.

\section{INTRODUCTION}

For Unmanned Underwater Vehicles (UUVs), power is the critical factor that often determines mission lifetime. Unlike many terrestrial vehicles, where batteries can be easily replaced or recharged, UUVs must either surface for battery replacement, or make watertight connections to safely recharge. In both cases, the solution typically requires human interaction, which increases the risk to both the vehicles and operators, as well as raising the total operating cost.

One solution for increasing the operational lifetime of UUVs is the use of inductive power transfer to wirelessly recharge the vehicle batteries while the UUV itself remains submerged. Inductive power transfer uses two electromagnetically coupled coils to transfer power from the transmitter (TX) to the receiver $(\mathrm{RX})$. For terrestrial applications, this type of power transfer is used for inductive stovetops and is currently being developed as a means to charge portable consumer electronics (cell phones, smart watches, etc.) and electric cars.

While the commercial protocols for wireless power transfer are useful for many consumer applications, there are a number of challenges that limit their use for UUV charging in the maritime environment. For example, the A4WP standard is based upon a power transmission frequency of $6.78 \mathrm{MHz}$. However, operating in salt water at this frequency leads to high attenuation and low power transfer efficiency. The Qi standard operates at a much lower frequency $(<250 \mathrm{KHz})$, but is currently limited to power levels on the order of $10 \mathrm{~W}$.
The development of a functional underwater wireless power transfer system for charging UUVs is a complex process and requires an examination of many factors, including biofouling, thermal dissipation, power transfer, and communications between the transmitter and receiver. This work focuses specifically on the wireless power transfer portion, and presents the fabrication and characterization of a power transfer system capable of delivering up to $75 \mathrm{~W}$ to the load. In this paper, the theory of underwater wireless power transfer is presented first. A high level overview of the system is provided next, followed by a detailed explanation of the coil and electronics design. The experimental setup used to demonstrate power transfer to a resistive load is then presented, followed by a discussion of the measured power transfer efficiency in both air and water. Finally, conclusions and future work are presented.

\section{DESIGN OF THE WIRELESS POWER TRANSFER SYSTEM}

A functional block diagram of the wireless power transfer system developed for this work is shown in Fig. 1. The transmit side of the system is comprised of a sinusoidal source, transmitter coil (TX), and tuning capacitor $\left(C_{T X}\right)$. For the characterization presented in this work, a function generator and linear amplifier were used to provide power for the transmitter. A more integrated solution of the transmitter circuitry is required for a functional implementation. The receive side of the system is made up
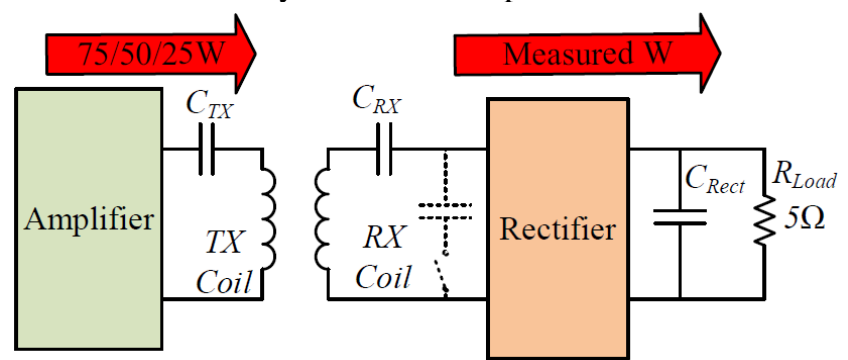

Fig -1: Block diagram of the wireless power transfer system.

of the receiver coil $(\mathrm{RX})$, tuning capacitor $\left(C_{R X}\right)$, rectification circuitry and a resistive load.

\subsection{WPT Coil Design}

For a set of coupled inductive coils, the efficiency of the wireless power transfer from the TX coil to the RX coil is given as, 


$$
\mu_{W P T}=\frac{k^{2} Q_{T X} Q_{R X}}{\left(1+\sqrt{1+k^{2} Q_{T X} Q_{R X}}\right)^{2}}
$$

Where $k$ is the coupling coefficient between the coils, and $Q_{T X}$ and $Q_{R X}$ are the quality factors of the TX and RX coils, respectively. To design a highly efficient system, both the coupling coefficient and quality factors should be maximized. The value of the coupling coefficient is based on the positioning of the coils (spacing and alignment) and the material between them (seawater), while the quality factors are related to the coils themselves (wire resistance, inductance and capacitance). For the planar coils implemented, it is desirable to align the coils as close together as possible so that the RX coil can capture as many of the flux lines emitted by the TX coil as possible. This means that the efficiency of the power transfer is highly dependent on the quality factors of the two coils.

For many terrestrial based systems, inductive power transfer coils are designed with peak $\mathrm{Q}$ values to occur in the $\mathrm{MHz}$ frequency range. While this practice is acceptable for terrestrial applications where air is the transfer medium, $\mathrm{MHz}$ frequencies do not propagate well underwater. Previous work in this area, however, has demonstrated that at short distances $(\leq 10 \mathrm{~cm})$ frequencies under $300 \mathrm{kHz}$ propagate in almost the same manner in water as they do in air. This means that power transfer coils can be designed and characterized to a first order in air with reasonable reliability at low frequencies.

The transfer efficiency for symmetrical coil is,

$$
\eta=\frac{k^{2} \omega^{2} L^{2}}{R^{2}+X^{2}+k^{2} \omega^{2} L^{2}}
$$

Where $\mathrm{k}$ is coupling coefficient and $\mathrm{R}, \mathrm{L}$ and $\mathrm{C}$ is Resistance, Inductance and Capacitance of the coil.

Which is when solved gives two equations. Trough equation and Ridge equation. For maximum efficiency, we only need ridge equation which is given by,

$$
R^{2}-X^{2}=k^{2} \omega^{2} L^{2}
$$

In this equation $\omega$ has two positive roots with same sign when discriminant is positive,

$$
\omega_{ \pm}=\left[\frac{\left(2-Q^{-2}\right) \pm \sqrt{\left(2-Q^{-2}\right)^{2}-4\left(1-k^{2}\right)}}{2\left(1-k^{2}\right)}\right]^{0.5} \omega_{0}
$$

The greater one $\omega_{+}$is the even splitting frequency and the smaller one $\omega_{\text {. }}$ is the odd splitting frequency.

\section{PROPOSED SYSTEM}

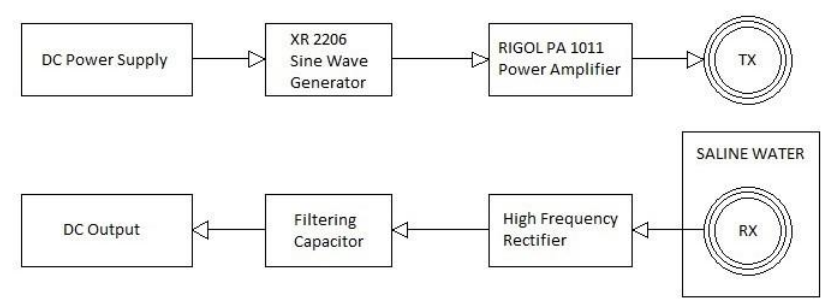

Fig-2: System Block Diagram

The system mainly consists of transmitter and receiver coil, dc power supply, sine wave generator, power amplifier, rectifier and filter circuit. Transmitter coil is fixed on a stand or a platform and may or may not be submerged on water. Receiver coil is placed on saline water. These 2 coils are positioned parallel to each other and on an axis passing through their center. High frequency current passing through transmitter produce magnetic flex which induce emf on receiver that can be used by the load.

\subsection{Coil Design}

\begin{tabular}{|l|l|}
\hline \multicolumn{1}{|c|}{ Parameter } & \multicolumn{1}{c|}{ Value } \\
\hline Coil inner Diameter & $50 \mathrm{~mm}$ \\
\hline Outer Diameter & $155 \mathrm{~mm}$ \\
\hline Wire Diameter & $2.3 \mathrm{~mm}$ \\
\hline Number of turns & 13 \\
\hline Inductance & $17.8 \mu \mathrm{H}$ \\
\hline Capacitance & $0.1 \mu \mathrm{F}$ \\
\hline
\end{tabular}

Table -1: Parameters of coils

Coil wire is made of multi-strand wire instead of single strand wire, since it has reduced skin effect and proximity effect losses at higher frequencies. The wire is made of 9 strands of 18 gauge wire which has approximate diameter of 12 gauge wire. Wires are wound on a plastic board. $0.1 \mu \mathrm{F}$ capacitor is connected in series to this coil giving resonant frequency at $119.29 \mathrm{kHz}$ according to inductance (L), capacitance (C) and frequency (f) relation,

$$
\begin{gathered}
f=\frac{1}{(2 \pi \sqrt{L C})} \\
f=\frac{1}{2 * \pi * \sqrt{17.8 * 10^{-6} * 0.1 * 10^{-6}}} \\
f=119.29 \mathrm{KHz}
\end{gathered}
$$

\subsection{Sine Wave Generator}

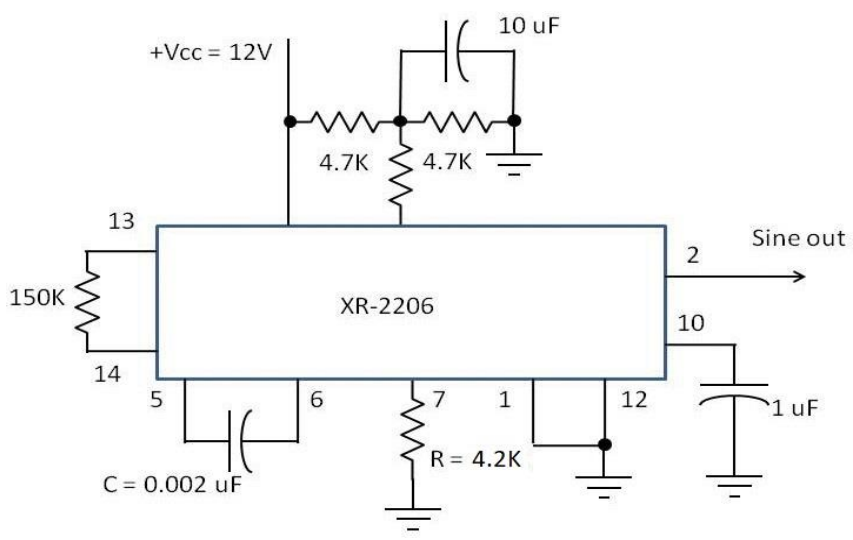

Fig-3: Sine Wave generator

The XR-2206 is a monolithic function generator IC that capable of producing high quality wave of high stability and accuracy. Capacitance between pin 5 and 6 and resistance between 7 and ground determines the frequency of sine wave generated. The frequency $\mathrm{f}$ is related to resistance $\mathrm{R}$ and capacitance $\mathrm{C}$ by the relation, 
$R=4.2 \mathrm{Kohm}$

$$
f=\frac{1}{R C}
$$

$C=2 n F$

Therefore,

$$
f=\frac{1}{4.2 * 10^{3} * 2 * 10^{-9}}
$$

This is close to target frequency $119.29 \mathrm{KHz}$.

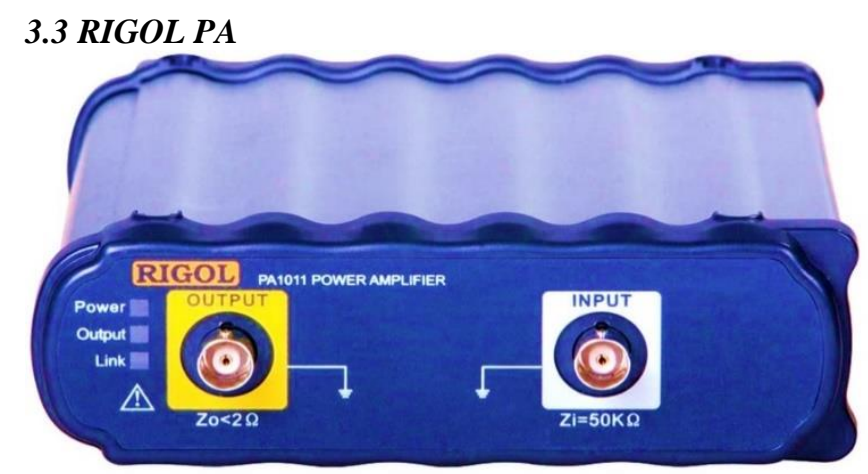

Fig-4: RIGOL Amplifier

The output from XR-2206 is amplified by PA $110125 \mathrm{~W}$ power amplifier produced by RIGOL Technology. It has up to $1 \mathrm{MHz}$ full bandwidth and higher than $80 \mathrm{~V} / \mu$ s slew rate.

\subsection{Transmission Medium}

Transmission is done on both air and saline water. So the parameters of both air and saline water need to be considered. Relative permeability $\left(\mu_{\mathrm{r}}\right)$ and relative permittivity $\left(\epsilon_{\mathrm{r}}\right)$ of air is unity. For Saline water relation between $\omega_{\mathrm{r}}$ and molarity (M) is given is figure 4.

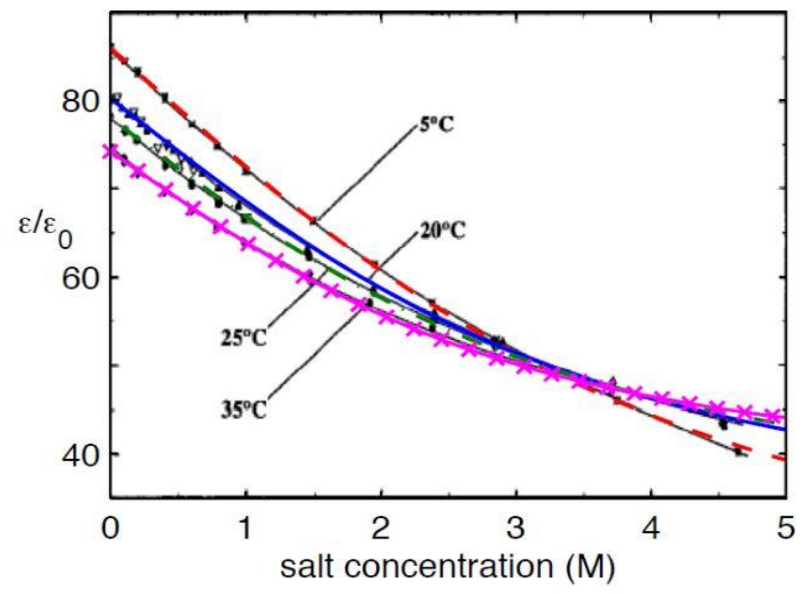

Fig-5: Relative Permittivity to Molarity plot

Relative permittivity $\left(\epsilon_{\mathrm{r}}\right)$ is inversely proportional to molarity (M). Normally seawater have $1 \mathrm{M}$ salt concentration. So the $\epsilon_{\mathrm{r}}$ is taken 60 for calculation. Relative permeability $\left(\mu_{\mathrm{r}}\right)$ of water is 1 . But for salt water it is somewhere between 0.9 and 1 . So $\mu_{\mathrm{r}}$ is taken 0.9 for calculation.

\section{CIRCUIT DIAGRAM}

Circuit is capable of transferring $25 \mathrm{~W}$ power at $119 \mathrm{KHz}$ frequency in both air and saline water. The transferred power is rectified and filtered to give DC out which can be used by UUV or any other underwater equipment.

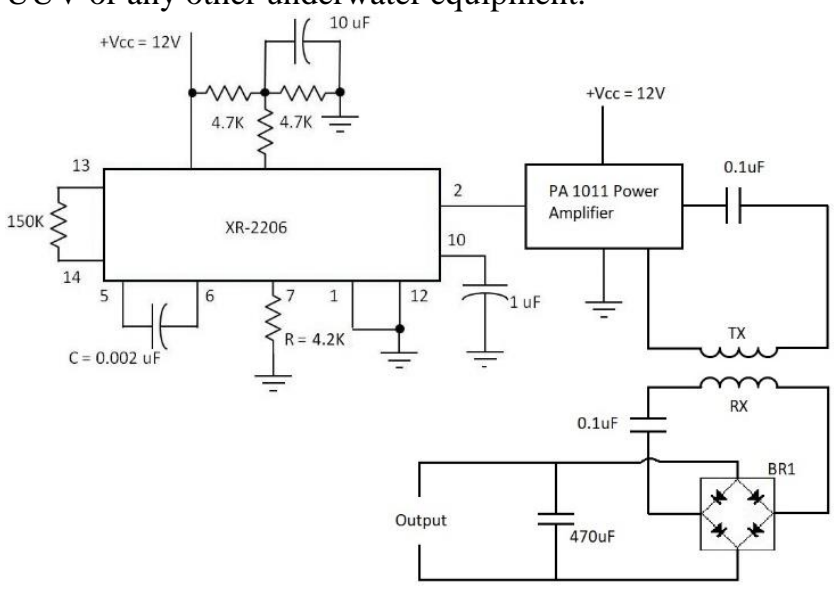

Fig-6: Designed Circuit

\section{SIMULATION}

Simulation of WPT system is done using MATLAB. All the simulation is done under the condition of saline water by giving 0.9 to relative permeability and 60 to relative permittivity.

Fig. 7 is the 3D simulation of Voltage, Distance and frequency.

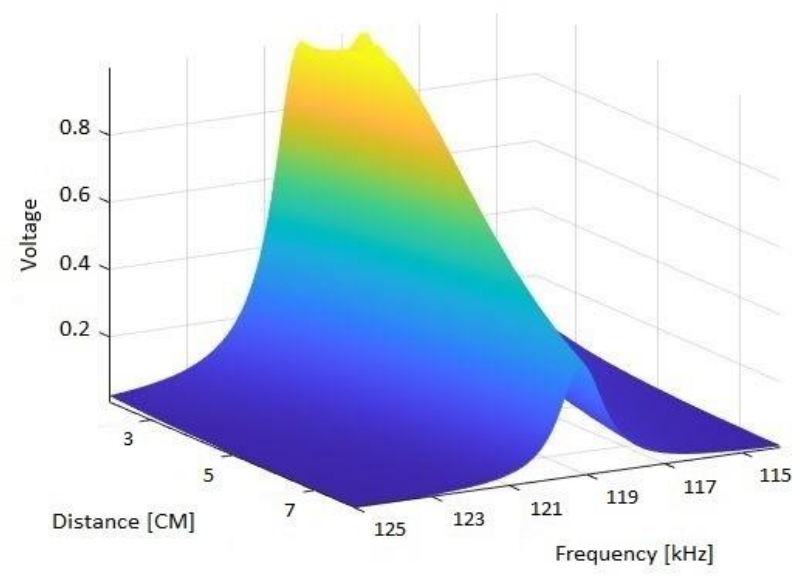

Fig-7: Frequency split graph

The maximum power transfer occur at distance $4 \mathrm{~cm}$. Frequency split is present in this distance interval causing maximum power transfer at 2 different frequencies other than resonant frequency.

Fig. 8 shows relation between coupling coefficient and distance. Red line represents saline water and blue line represents air. As distance decreases coupling coefficient increases but never reaches 1 because of frequency splitting. 


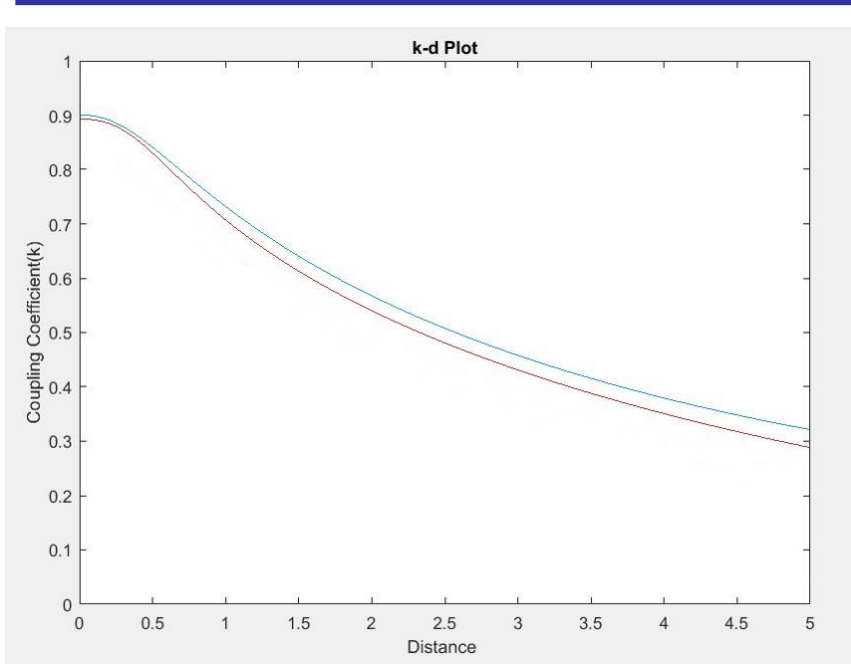

Fig-8: coupling coefficient - distance graph

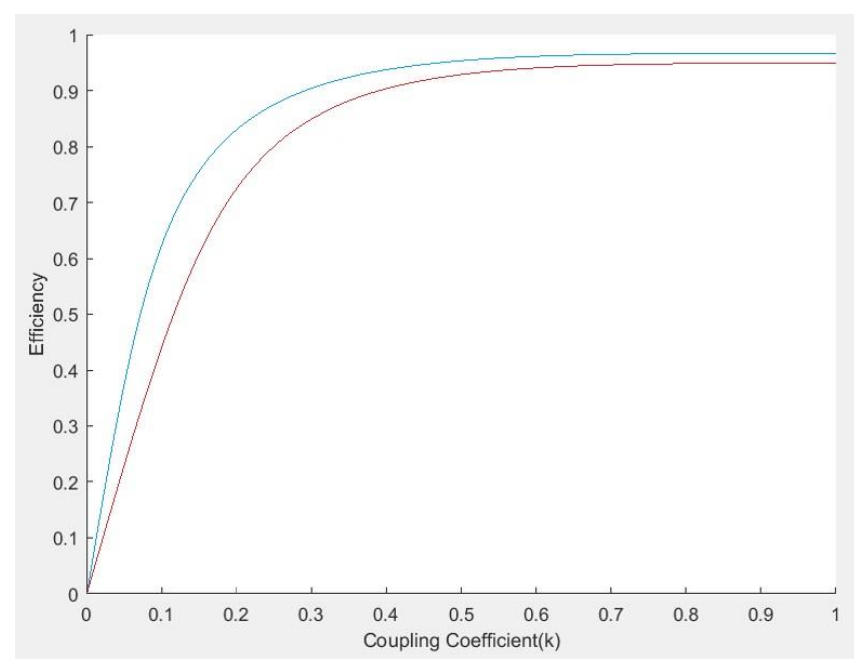

Fig-9: Efficiency Plot

Fig. 9 shows relation between coupling coefficient and efficiency. From Fig. 8 coupling coefficient for this distance is 0.35 and efficiency for this value from fig. 8 is around $85 \%$.

\section{CONCLUSION}

The design and simulation of a wireless power transfer system for underwater applications is presented. We have demonstrated the ability to model the wireless transfer of power and shown that at frequencies below $250 \mathrm{KHz}$, there is little difference between transferring power between saline water and air. No significant differences of power transfer of this planar two-coil WPT system are observed in air and seawater in this frequency. Maximum of $85 \%$ transfer efficiency is possible at a distance of $4 \mathrm{~cm}$. larger diameter coils of same inductance could increase the maximum power transferring distance.

\section{REFERENCES}

[1] Bana V., Kerber M., Anderson G., et al.: "Underwater wireless power transfer for maritime applications". IEEE Wireless Power Transfer Conf. (WPTC), 2018.

[2] Wangqiang Niu, Wei Gu, Jianxin Chu, "Analysis and experimental results of frequency splitting of underwater wireless power transfer", Eng, 2017, vol. 2017, iss. 7, pp. 385-390.

[3] Han J., Asada A., Ura T., "Noncontact power supply for seafloor geodetic observing robot system", J. Mar. Sci. Technol., 2007, pp. 183-189.

[4] Itoh R., Sawahara Y., Ishizaki T., "Wireless power transfer to moving ornamental robot fish in aquarium", 2014 IEEE 3rd Global Conf. Consumer Electronics.

[5] Niu W., Gu W., Chu J., "Frequency splitting patterns in wireless power relay transfer", IET Circuits Devices syst., 2014, 8, (6), pp. 561-567.

[6] Fukuda H., Kobayashi N., Shizuno K., et al.: "New concept of an electromagnetic usage for contactless communication and power transmission in the ocean". IEEE Int. Underwater Technology Symp. (UT), 2013.

[7] Niu W., Gu W., Chu J., et al.: "frequency splitting of underwater wireless power transfer". IEEE Int. Workshop on electromagnetics, Nanjing, China, 2016.

[8] Boroujeni S.R., Shahabadi M., Rashed-Mohassel J.: "Investigation of electric and magnetic coupling between two helical resonators of a wireless power transfer system", Electron. Lett., 2016. 